\title{
Effects of Maryland's law banning Saturday night special handguns on crime guns
}

\author{
Jon S Vernick, Daniel W Webster, Lisa M Hepburn
}

\begin{abstract}
Objectives-To determine the effects of a 1988 Maryland law that banned "Saturday night special" handguns on the types of guns used in crime. To determine if controls on the lawful market for handguns affect the illegal market as well. Setting-Baltimore, Maryland, and 15 other US cities participating in a crime gun tracing project.

Methods-Cross sectional comparison of the proportion of crime guns that are banned by the Maryland law, comparing Baltimore, MD with 15 other cities outside of Maryland. Multivariate linear regression analysis to determine if observed differences between Baltimore and 15 other cities are explained by demographic or regional differences among the cities rather than Maryland's law.

Results-Among crime guns, a gun banned by Maryland's law is more than twice as likely (relative risk (RR) $2.3,95 \%$ confidence interval (CI) 2.0 to 2.5 ) to be the subject of a crime gun trace request in 15 other cities combined, than in Baltimore. Among homicide guns, a crime especially relevant for public safety, a comparable difference (RR 2.1, 95\% CI 1.1 to 4.2) was observed. The proportion of Baltimore's crime guns that are banned is 12 percentage points lower than would be expected based on its demographic and regional characteristics alone. Among crime guns purchased after 1990, a much smaller proportion in Baltimore are banned models than in 15 other cities. Conclusions-Maryland's law has reduced the use of banned Saturday night specials by criminals in Baltimore. Contrary to the claims of some opponents of gun control laws, regulation of the lawful market for firearms can also affect criminals.

(Injury Prevention 1999;5:259-263)
\end{abstract}

Keywords: firearms; evaluation; gun control; Saturday night special

Do gun control laws aimed at regulating the lawful purchase of firearms affect only law abiding citizens, or are criminals also affected? This is a central, though largely unanswered, question for gun policy. Pro-gun advocacy organizations argue that criminals, by definition, don't obey laws and so restrictions on gun purchase or possession will only inconvenience the law abiding. ${ }^{1}$ Others have reasoned that controls on the lawful firearms market may increase the real "cost" paid by criminals to obtain a firearm through illicit means. ${ }^{2}$ If at least some criminals are sensitive to these changes in the difficulty of obtaining a gun, they may be less able or willing to obtain one unlawfully. Only if this latter argument is accurate, can gun control laws reduce the likelihood of injury or death associated with the criminal use of guns.

One gun control measure that continues to receive attention in the US and in other nations is to ban specific categories of firearms deemed especially dangerous or attractive to criminals. For example, Australia recently banned certain rifles and shotguns, ${ }^{3}$ Great Britain outlawed most handguns, ${ }^{4}$ and Canada prohibits assault weapons and certain handguns. ${ }^{5}$

As early as the 1960 s, the gun policy debate in the US began to include a focus on one such class of firearms, so-called "Saturday night specials"-handguns that have been described as unreliable, inaccurate, poorly made, easily concealable, inexpensive, and disproportionately involved in crime. ${ }^{6}$ In 1968, the US Congress passed the Gun Control Act. Among the Act's numerous provisions is a ban on the importation of handguns deemed not "particularly suitable for or readily adaptable to sporting purposes". ${ }^{7}$ Regulations implementing this language had the effect of outlawing the importation of most small, poorly made handguns. However, the law allowed the manufacture and sale of domestic guns to proceed largely unchecked. Today a number of manufacturers located in southern California, described collectively by Wintemute as the "ring of fire", have filled this market niche. ${ }^{8}$

Without federal law regulating the domestic manufacture and sale of these guns, several states have passed laws to ban Saturday night specials. On 23 May 1988, Maryland became the first state since 1975 to pass a law intended to ban the manufacture and sale of Saturday night specials. ${ }^{910}$ The law established a Handgun Roster Board empowered to compile a list of handguns approved for manufacture and sale in Maryland. The law requires the board to consider nine factors in determining whether a 
gun is approved for sale, including its concealability, quality, reliability, and utility for legitimate uses. ${ }^{11}$ As of 1 January 1990, no handgun manufactured after 1 January 1985 can be sold in Maryland unless it specifically appears on the handgun roster of approved guns.

Recently in the US, there has been renewed interest in regulating Saturday night specials. Under newly coined terms like "junk guns", legislative proposals in Congress would require domestic guns to meet the same standards as imported ones. ${ }^{12}$ California ${ }^{13}$ and Massachusetts $^{14}$ recently enacted new state laws to outlaw Saturday night specials.

Yet the effect of Saturday night special bans on the type of guns chosen by criminals has never been assessed. Recent research, however, has demonstrated that persons with a criminal history were more likely to purchase or attempt to purchase small, inexpensive handguns than those without a criminal history. ${ }^{15}$ If some criminals indeed favor specific kinds of guns, perhaps those preferences are resistant to change and criminals will aggressively seek out those gun models through illegal channels even if they are banned. Conversely, a law targeting the lawful market for a specific class of handguns, like Saturday night specials, might successfully disrupt the unlawful market as well, and affect the use of these guns by criminals. Therefore, understanding how a ban on Saturday night specials affects the types of guns used by criminals is an important first step to understanding the law's ability to affect injuries and deaths associated with violent crime.

This study of Maryland's law banning Saturday night special handguns will (1) identify the proportion of all crime guns that are banned by the law, comparing Baltimore, Maryland with 15 other cities outside of Maryland that do not ban Saturday night specials, and (2) include multivariate and longitudinal analyses to assess whether observed differences between Baltimore and these others cities are, in fact, attributable to Maryland's law.

\section{Methods}

DATA SOURCE

Our analyses of the specific gun models used in crime relies on firearm tracing data compiled by the US Bureau of Alcohol, Tobacco, and Firearms (ATF). At the request of law enforcement officers, the ATF will trace firearms from their manufacturer to their first retail purchaser. In most cities and states, only a subset of all crime guns are traced, so analyses of specific gun models used in crime are subject to selection bias (PH Blackman. Paper delivered at Homicide Research Working Group Spring Symposium, Shepherdstown, WV, June 1997). However, in July 1996 the ATF began a project known as the Youth Crime Gun Interdiction Initiative (YCGII). Under the YCGII, 17 US cities, including Baltimore, Maryland, agreed to submit trace requests for all crime guns, defined as "any firearm that is illegally possessed, used in a crime, or suspected by law enforcement officials of being used in a crime". ${ }^{17}$ The 17 cities are geographically diverse, representing 15 states and Washington, DC. Despite its name, the initiative was not limited to tracing guns associated with juvenile offenders. YCGII data were available for the first phase of that project, the 10 month period from 1 July 1996 to 30 April 1997.

Most of our analyses rely on trace requests, rather than completed traces. This eliminates another potential source of bias, if some gun models are more likely to be successfully traced to their purchasers. In one of the 17 cities, Washington, DC, most private ownership of handguns has been banned since 1976. For this reason, we excluded Washington from our analyses. None of the remaining 16 cities, other than Baltimore, bans Saturday night specials or all handguns.

\section{BIVARIATE ANALYSES}

We analyzed YCGII data to determine what proportion of the crime guns in Baltimore were among those banned by the Maryland law, compared with the other cities represented. We determined if a specific gun model was banned in Maryland, or not, as follows: (1) If the YCGII dataset included manufacturer, model, and caliber information for a given gun, we consulted the Maryland handgun roster; guns that do not appear on the roster are banned. (2) If the dataset included only the manufacturer and caliber, but not the model, for a given gun, we consulted gun publications to determine which model(s) that manufacturer produced. ${ }^{18}$ If the manufacturer produced only one handgun model in that caliber, or if all of that manufacturer's handgun models within a given caliber appeared on the handgun roster, then we could determine the gun's legal status. Otherwise, or if basic manufacturer information was missing, we placed that gun into the unknown category. (3) The dataset identifies long guns (that is, rifles and shotguns) all of which are unaffected by Maryland's 1988 law. In this way, we were able to categorize all but $1311(3.5 \%)$ of 37439 guns in the dataset.

Most guns banned by Maryland's law were of low caliber. Therefore, we examined whether the law may have led to relatively more medium and high caliber guns being used in crime in Baltimore compared with the other cities. Following other research, small caliber was defined as .22, .25, and .32; medium as .38, .380 , and $9 \mathrm{~mm}$; and large as .357, .40, .44, .45 , and $10 \mathrm{~mm} \cdot{ }^{15}$ Long guns were categorized separately.

Maryland's law bans most, but not all, inexpensive, low quality handguns, and we hypothesized that some price sensitive consumers may have been particularly inclined to seek out these specific non-banned guns. To determine if specific handguns not banned by Maryland's law were more prevalent among Baltimore's crime guns, we compared the proportion of likely "substitution" guns among the cities in the dataset. We defined substitution handguns as those (1) not banned by the Maryland law and (2) with a suggested retail price of $\$ 150$ or less in $1996 .^{18}$

Because homicide is the most completely reported crime, we also compared the pro- 
Table 1 Crime gun trace requests and relative risks for guns banned by 1988 Maryland (MD) law, in Baltimore and other regions, 1 fuly 1996 to 30 April 1997

\begin{tabular}{lccll}
\hline City/region & $\begin{array}{l}\text { All gun trace } \\
\text { requests }\end{array}$ & $\begin{array}{l}\text { No of guns } \\
\text { banned by } 1988 \\
\text { MD law }\end{array}$ & $\begin{array}{l}\text { \% Of guns } \\
\text { banned by 1988 } \\
\text { MD law }\end{array}$ & $\begin{array}{l}\text { Relative risk } \\
\text { (confidence } \\
\text { interval) }\end{array}$ \\
\hline Baltimore & 3596 & 314 & 8.7 & 1.0 \\
15 Other cities† & 30623 & 6036 & 19.7 & $2.3(2.0$ to 2.5) \\
Northeast & 14042 & 3060 & 21.7 & $2.5(2.3$ to 2.8) \\
Southฐ & 6114 & 1457 & 23.8 & $2.7(2.5$ to 3.0) \\
Central $\|$ & 6908 & 995 & 14.4 & $1.7(1.5$ to 1.9$)$ \\
West & 3559 & 524 & 14.7 & $1.7(1.5$ to 1.9$)$ \\
\hline
\end{tabular}

${ }^{\star}$ Excludes guns whose banned or non-banned status could not be determined.

†Atlanta GA, Birmingham AL, Boston MA, Bridgeport CT, Cleveland OH, Inglewood CA, Jersey City NJ, Memphis TN, Milwaukee WI, New York NY, Richmond VA, Salinas CA, San Antonio TX, Seattle WA, St Louis MO.

‡Boston, Bridgeport, Jersey City, New York.

SAtlanta, Birmingham, Memphis, Richmond.

$\|$ Cleveland, Milwaukee, St Louis.

Inglewood, Salinas, San Antonio, Seattle.

portion of banned guns among the subset of those crime guns linked with a homicide.

MULTIVARIATE AND LONGITUDINAL ANALYSES

The YCGII does not provide information for crime guns seized before the enactment of Maryland's law in 1990. Therefore, it is possible that any observed differences between Baltimore and the remaining cities, in the proportion of crime guns that are banned, might be explained by pre-existing differences among the cities themselves, and not by the effect of Maryland's law in Baltimore. To examine this hypothesis, we estimated a multiple linear regression model for the per cent of crime guns in a city that are banned under Maryland's law. Potential covariates were chosen to reflect city level demographic characteristics that might affect decisions to purchase small, inexpensive guns rather than other guns. These included the per cent of adults with income below the federal poverty line, the per cent of a city's population who were age 15-24 (since youth may be more price sensitive than adults), and the per cent who were black males (since this group is over-represented among firearm crimes, such as robbery, that might be associated with small, concealable guns). ${ }^{19}$ These demographic data were obtained from the 1990 census. The model also includes a dummy variable to reflect the effect of Maryland's law in Baltimore, and a regional dummy variable to capture any unmeasured regional characteristics. The dummy variables are set equal to 1 where the law or region is present, and zero otherwise. Dummy variables are commonly used in regression analyses evaluating gun laws. ${ }^{21}$

As an additional effort to determine if observed differences between Baltimore and other cities are attributable to Maryland's law, we examined the year of purchase for the subset of crime guns for which this information was available. For each year from 1985 to 1997, we determine the proportion of guns purchased in that year that were banned by Maryland's law and that were used in crime during the study period (1 July 1996 to 30 April 1997). If Maryland's law affects which guns criminals choose, one would expect that Baltimore's crime guns purchased after 1 January 1990 will be less likely to be Saturday night specials than in other cities. Of crime guns purchased before 1990, however, the pro- portion in Baltimore that are Saturday night specials should be more comparable to the proportion in the 15 other cities. We limit this analysis to guns purchased from 1985 to 1997 because of the very small number of 1996-97 crime guns purchased before 1985 .

\section{Results}

BIVARIATE ANALYSES

As of December 1996, more than 1000 different handgun models appear on the handgun roster of approved guns. At the same time, more than 100 different handgun models have been banned by Maryland's law, either because they have been specifically disapproved by the Handgun Roster Board, or because they simply do not appear on the list of approved guns.

Excluding Washington, DC, there were 34219 guns in the YCGII dataset whose banned or non-banned status could be determined. Of these, 6350 (18.6\%) were banned by Maryland's 1988 law.

Among crime guns, guns banned by Maryland's law were more than twice as likely (relative risk (RR) 2.3, 95\% confidence interval (CI) 2.0 to 2.5 ) to be the subject of a trace request in the 15 other cities combined $(19.7 \%$ of crime guns) than in Baltimore $(8.7 \%$ of crime guns); see table 1 . When we divided the 15 cities into four geographic regions, guns banned by the Maryland law were significantly more likely to appear in each region than in Baltimore, indicating that the overall result is not unduly affected by the results for any one city. The difference was greatest for the eight cities in the northeast and south, regions that are arguably most comparable to Baltimore.

The crimes represented by the YCGII data include violent crimes, property crimes, and illegal gun possession. If only guns associated with a homicide are considered, banned guns are still more than twice as likely to be the subject of a trace request in the 15 other cities combined than in Baltimore (RR 2.1, 95\% CI 1.1 to 4.2 ).

We identified six handguns that met our definition of a substitution gun. Most numerous among these non-banned guns were the Davis P32, Davis P38, and Phoenix HP22. Others included inexpensive handgun models made by HiPoint, New England Firearms, and Stallard Arms. Taken together, the six nonbanned guns were somewhat less likely (RR $0.8,95 \%$ CI 0.7 to 0.9 ) to be among the crime guns in the 15 other cities ( $5.5 \%$ of crime guns) than in Baltimore $(6.9 \%)$. If banned guns and these six non-banned guns are considered together, however, they represent $15.7 \%$ of crime guns in Baltimore, compared with $25.2 \%$ in the other 15 cities combined (RR 1.6, $95 \%$ CI 1.5 to 1.7$)$. Thus, the reduction in the proportion of banned guns among Baltimore's crime guns is larger than any substitution effect to these six non-banned guns.

Similarly, there was no evidence of meaningful substitution to larger caliber handguns in Baltimore compared with the 15 other cities. In Baltimore, of 3757 guns for which caliber information was available 1048 (27.9\%) were 
Table 2 Linear regression on the per cent of crime guns (fuly 1996 to April 1997) banned by 1988 Maryland law

\begin{tabular}{lccc}
\hline Explanatory variable & Coefficient & $\begin{array}{l}\text { Standard } \\
\text { error }\end{array}$ & p Value \\
\hline \% Of adults below poverty line & 0.58 & 0.54 & 0.31 \\
\% Of city population that are black males age 15-24 years & 0.41 & 1.01 & 0.70 \\
Regional dummy variable (north, south = 1) & 4.71 & 2.32 & 0.07 \\
Baltimore dummy variable (Baltimore = 1) & -12.66 & 4.20 & 0.01 \\
Constant & 7.09 & 5.50 & 0.22
\end{tabular}

$R^{2}=0.61$.

small caliber, $1820(48.4 \%)$ were medium or large caliber, and $889(23.7 \%)$ were long guns. The comparable percentages for 31349 guns in the 15 other cities were: $24.4 \%$ small caliber, $56.0 \%$ medium or large, and $19.6 \%$ long guns.

MULTIVARIATE AND LONGITUDINAL ANALYSES

The results of the multiple linear regression analysis are displayed in table 2 . The regression coefficient for the dummy variable representing the effect of Maryland's law $(\beta=-12.66)$ indicates that the per cent of Baltimore's crime guns that are banned is about 12 percentage points lower than would be expected based on its demographic and regional characteristics. This is comparable to the crude difference of 11 percentage points between Baltimore $(8.7 \%)$ and the 15 other cities $(19.7 \%)$ in table 1. Therefore, the observed differences between Baltimore and the other cities are not substantially a function of any city level demographic differences included in the model. With only 16 observations of the outcome measure, however, the number of explanatory variables that can be included is necessarily limited. Nevertheless, the model fit is relatively good $\left(R^{2}=0.61\right)$.

Of the 3596 guns used in crime in Baltimore from 1 July 1996 to 30 April 1997, purchase year information is available for 1143 guns, 1092 of which were purchased from 1985 to 1997. For the 15 other cities, 9490 traceable crime guns were bought during that same time period. Figure 1 plots the proportion of crime gun models purchased in a given year that were banned by the Maryland law. It is important to recognize that all of these guns were associated

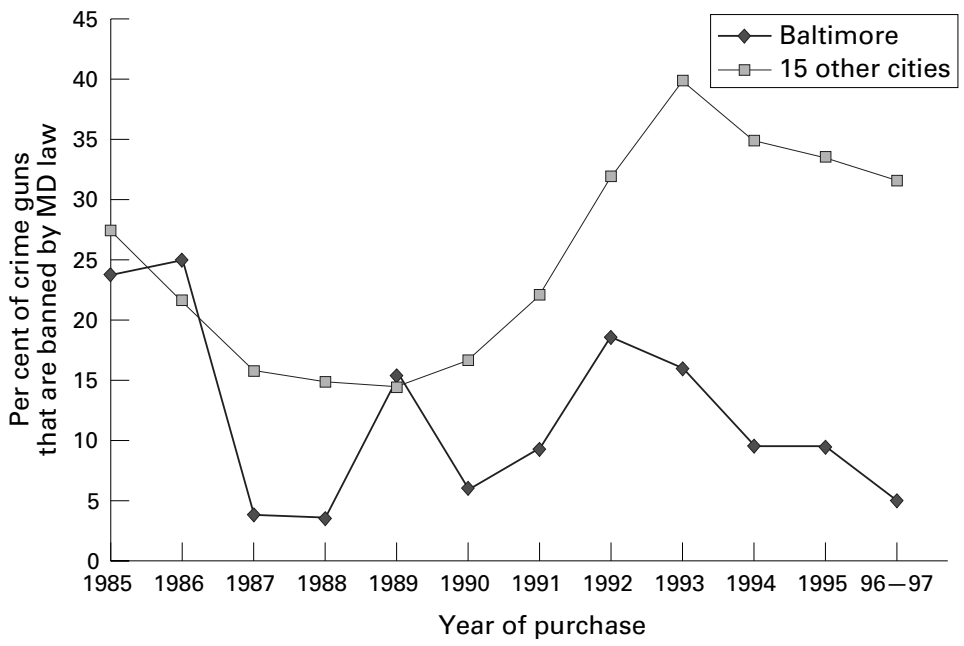

Figure 1 Per cent of guns used in crime from fuly 1996 to April 1997 that are banned by Maryland law (effective 1 fanuary 1990) by year of gun purchase. with a crime in the 1996-97 study period; only their year of first purchase varies.

Taken together, for guns used in crime in 1996-97 in Baltimore, $13.6 \%$ of those initially purchased from 1985-89 were banned Saturday night specials, compared with $17.6 \%$ in the 15 other cities. For guns purchased from 1990 to 1997 (after Maryland's law became effective), however, the difference between Baltimore and the 15 other cities is much larger: $10.4 \%$ of crime guns in Baltimore are banned models, compared with $32.4 \%$ in the 15 other cities.

\section{Discussion}

The proportion of all crime guns that are banned gun models is significantly smaller in Baltimore than in 15 other cities. Multivariate and longitudinal analyses suggest that this difference is likely to be attributable to Maryland's law banning Saturday night special handguns. By banning these guns in the lawful market, Maryland appears to have affected the type of gun used in crime. This belies the argument, often raised by opponents of stricter regulation of firearms, that only law abiding citizens, and not criminals, are affected by gun control laws. The consistency of the results among the subset of guns associated with a homicide suggests that these findings are highly relevant for public safety. In fact, other research has demonstrated that one of the guns banned by Maryland's law, the Raven MP-25, was the most frequently identified handgun among firearm related homicides and suicides in one city without a ban. ${ }^{22}$

One concern regarding Saturday night special bans is that criminals will simply substitute similar or even larger caliber guns for those that are banned. Our data do not provide strong support for this argument. Although inexpensive, non-banned handguns were somewhat more likely to be among the crime guns in Baltimore compared with the other 15 cities; this effect is modest compared with the effect of the law on banned guns. Similarly, despite making generally low caliber Saturday night specials less common among Baltimore's crime guns, in 1996-97 the proportion of crime guns that were medium or large caliber was actually lower in Baltimore than in the 15 other cities combined.

There are several possible limitations to our analyses. As we have indicated, reliable tracing data were not available for guns used in crime before the law was implemented. Differences in the proportion of crime guns that are Saturday night specials, therefore, could represent baseline differences that were unaffected by the law. However, our multivariate analysis, controlling for demographic and regional differences among the cities, found that the variable associated with Maryland's law explained the largest share of the variance among the cities. In addition, among the 1996-97 crime guns purchased before the implementation of Maryland's law in 1990, the baseline proportion that were Saturday night specials was quite comparable in Baltimore and the 15 other cities. 
Among crime guns purchased after 1990, however, Baltimore has a substantially lower proportion of Saturday night specials among its crime guns than in the other cities.

Despite their agreement to trace all crime guns, some cities may have more completely implemented this program than others. But such differences should only affect cross sectional comparisons of the proportion of crime guns that are Saturday night specials if some jurisdictions are more likely to choose specific gun models to trace. The consistency and magnitude of our findings across cities and regions of the country, however, should diminish concern about this possible bias.

Since 1990, there has been a shift in the US handgun market toward larger caliber pistols. ${ }^{23}$ This may mean that the proportion of crime guns in Baltimore that are lower caliber Saturday night specials would have declined since 1990 even without the new law. But this national trend in the handgun preferences of consumers should have affected the cities in our analysis in roughly the same waytherefore, the relative difference between Baltimore and those other regions should be unaffected by these national trends.

\section{Implications for prevention}

Understanding how Maryland's law has affected more intermediate outcomes, such as the types of guns use in crime, can provide an important lesson to other jurisdictions considering similar laws. Banning a specific class of handguns, in this case Saturday night specials, can affect the type of gun used by criminals. For countries that have enacted or are considering banning even larger categories of firearms, such as all handguns, these results suggest that criminal use of the banned guns may also be dramatically reduced. But only if substitution to equally lethal weapons is limited, will such laws influence more distal outcomes, like homicide rates.
This research was supported by grant R49CCR302486 from the Centers for Disease Control and Prevention to the Johns Hopkins Center for Injury Research and Policy, and by a grant from the Joyce Foundation, Chicago, Illinois to the Johns Hopkins Center for Gun Policy and Research.

1 National Rifle Association. The NRA member guide. Washington, DC: National Rifle Association, 1992.

2 Cook PJ. Strategic thinking about gun markets and violence. 7AMA 1996;275:1765-7.

3 Peters R, Watson C. A breakthrough in gun control in Australia after the Port Arthur massacre. Inj Prev 1996;2:253-

4 Associated Press. Handgun ban in Britain passes crucial test. New York Times 12 June 1997: A15.

5 Cukier W. Firearms regulations: Canada in the international context. Chronic Dis Can 1998;19:25-34.

6 Sherrill R. The Saturday night special. New York, NY: Charterhouse, 1973.

7 Gun Control Act of 1968, $₫ 925$ (d) (3).

8 Wintemute GJ. Ring of fire: the handgun manufacturers of Southern California. Sacramento, CA: Violence Prevention Research Program, 1994.

9 MD. Code Ann. \$36J(b)(1)

10 Teret SP, Alexander GR, Bailey LA. The passage of Maryland's gun law: data and advocacy for injury Maryland's gun law: data and advocacy for

prevention. F Public Health Policy 1996 (i)
1 MD. Code Ann. \$36J(b)(2) (ix).

12 American Handgun Standards Act, S 70, 105th Congress (1997).

13 Williams DA. California gun controls signed into law. Associated Press 28 August 1999.

14 Gun Control Act of 1998, Massachusetts St 1998, c180, $\$ 47$.

15 Wintemute GJ, Parham CA, Wright MA, et al. Weapons of choice: previous criminal history, later criminal activity, and firearm preference among legally authorized young adult purchasers of handguns. F Trauma 1998;44:155-60.

16 Wintemute GJ, Wright MA, Parham CA, et al. Denial of handgun purchase: a description of the affected population and a controlled study of their handgun preferences. Fournal of Criminal fustice 1999;27:21-31.

17 Bureau of Alcohol, Tobacco, and Firearms. Crime gun trace analysis reports: the illegal youth firearm markets in 17 communities. Washington, DC: US Government Printing Office, 1997.

18 Warner K, ed. Gun digest. Iola, WI: DBI Books, 1997.

19 Federal Bureau of Investigation. Uniform crime reports for the United States, 1997. Washington, DC: US Department of Justice, 1998

20 Cummings P, Grossman DG, Rivara FP, et al. State gun safe storage laws and child mortality due to firearms. $f A M A$ 1997;278:1084-6.

21 Loftin C, McDowall D, Wiersema B, et al. Effects of restrictive licensing of handguns on homicide and suicide in the District of Columbia. N Engl f Med 1991;325:1615-20.

22 Hargarten SW, Karlson TA, O'Brien M, et al. Characteristics of firearms involved in fatalities. $\mathcal{F} A M A$ 1996;275:4-5.

23 Wintemute GJ. The relationship between firearm design and firearm violence: handguns in the 1990s. FAMA 1996; 275:1749-53. 